\title{
MAXIMUM PRINCIPLES FOR VECTORIAL APPROXIMATE MINIMIZERS OF NONCONVEX FUNCTIONALS
}

\author{
NIKOLAOS I. KATZOURAKIS
}

\begin{abstract}
We establish Maximum Principles which apply to vectorial approximate minimizers of the general integral functional of Calculus of Variations. Our main result is a version of the Convex Hull Property.

The primary advance compared to results already existing in the literature is that we have dropped the quasiconvexity assumption of the integrand in the gradient term. The lack of weak Lower Semicontinuity is compensated by introducing a nonlinear convergence technique, based on the approximation of the projection onto a convex set by reflections and on the invariance of the integrand in the gradient term under the Orthogonal Group.

Maximum Principles are implied for the relaxed solution in the case of nonexistence of minimizers and for minimizing solutions of the Euler-Lagrange system of PDEs.
\end{abstract}

\section{INTRODUCTION.}

Let $\mathscr{L}: \Omega \times \mathbb{R}^{N} \times \mathbb{R}^{N \times n} \longrightarrow \mathbb{R}$ be a Carathéodory function where $\Omega \subseteq \mathbb{R}^{n}$ is an open set. In this paper we are concerned with the derivation of Maximum Principle results applying to Approximate Minimizers of the functional

$$
E(u, \Omega):=\int_{\Omega} \mathscr{L}(x, u(x), D u(x)) d x
$$

placed in $\left[W_{g}^{1, q}(\Omega)\right]^{N}, q \geq 1$ with prescribed boundary values $g \in\left[W^{1, q}(\Omega)\right]^{N}$. A vector function $u: \Omega \subseteq \mathbb{R}^{n} \longrightarrow \mathbb{R}^{N}$ will be called an $\alpha$-Minimizer of (1.1) if for some $\alpha \geq 0$ and all $\phi \in\left[W_{0}^{1, q}(\Omega)\right]^{N}$, we have

$$
E(u, \Omega) \leq E(u+\phi, \Omega)+\alpha .
$$

Minimizing families $\left\{u_{\alpha}\right\}_{\alpha>0}$ correspond to minimizing sequences as $\alpha \rightarrow 0$ of the variational problem

$$
E(u, \Omega)=\inf _{\left[W_{g}^{1, q}(\Omega)\right]^{N}} E
$$

and 0-minimizers correspond to solutions of (1.3). Our viewpoint of the Maximum Principle in the vector case is based on the observation that the scalar inequalities $\sup _{\Omega} u \leq \max _{\partial \Omega} u, \inf _{\Omega} u \geq \min _{\partial \Omega} u$ when $N=1$ can be recast as $u(\Omega) \subseteq\left[\min _{\partial \Omega} u, \max _{\partial \Omega} u\right]$. When $N \geq 1$, the appropriate vectorial extension is the so-called Convex Hull Property

$$
u(\Omega) \subseteq \overline{\mathrm{co}}(u(\partial \Omega))
$$

Key words and phrases. Maximum Principle, Convex Hull Property, Calculus of Variations, Minimizers, Nonconvex functionals, Relaxation, Euler-Lagrange system of PDEs. 
and states that the range is contained in the closed convex hull of the boundary values.

Maximum Principles either as norm bounds or in the form (1.4) applying to the functional (1.1) and its minimizing solutions of the respective Euler-Lagrange system of PDEs are a well-established subject and provide a priori localization and bounds, necessary for further regularity investigations. Without attempting to provide a complete list of papers, we refer to Alexander-Ghomi [AG], Bildhauer-Fuchs [BF1], [BF2], Colding-Minicozzi II [CM], D'Ottavio-Leonetti-Musciano [DLM], Leonetti [L1], [L2], Leonetti-Siepe [LS] and Osserman [O1], [O2].

In this paper we derive appropriate versions of the Convex Hull Property (1.4) for approximate minimizers of the functional (1.1). These results are inherited by the relaxed solutions of (1.3) and by minimizing solutions of the Euler-Lagrange system of PDEs, in case they exist.

Our primary advance compared to similar results existing in the literature is that we have dropped the quasiconvexity assumption of $\mathscr{L}$ in the gradient term. This is achieved by introducing a nonlinear approximation technique which establishes (1.4) without invoking the weak Lower Semi-Continuity of (1.1). The essential ingredients are the construction of systematic approximations of the projection map onto convex sets generated by reflections and some (almost) invariance of $P \mapsto$ $\mathscr{L}(x, \eta, P)$ under the Orthogonal Group of $\mathbb{R}^{N}$. The latter is automatically satisfied if $\mathscr{L}=\mathscr{L}(x, \eta,|P|)$.

Our approximations of the projection map by reflections, the "Folding Maps", are employed to construct suitable energy comparison functions. Roughly, by projecting the minimizer on the convex hull of its boundary values we would get an energy-decreasing deformation. However, in the absence of convexity by projecting we may not decrease the energy. We obtain our deformations as energy-preserving piecewise isometries which converge to the projection.

A subtle point of the nonconvex case is that we can assert a version of (1.4) only for at least one approximate minimizer among all that may exist. This allows to utilize (1.4) as a Selecting Principle in the sense of Dacorogna-Ferriero [DF] to rule out non-physical solutions. Moreover, our approximation method is fairly general and could be useful in other contexts as well. For this reason, Section 2 which is devoted to the construction of the approximations is independent of Section 3 in which the approximations are employed to derive our Maximum Principles. We also note that our results in principle could be useful to nonconvex problems arising in the nonlinear theory of Elasticity [B].

The main ideas arising in this work is an outgrowth of methods employed as stepping stones in order to solve the problem studied in [AK], by Alikakos and the Author. The choice to formulate our results for approximate minimizers is essential; in the absence of quasiconvexity, existence of minimizers is not to be expected. Moreover, approximate minimizers satisfy a locality property which is not enjoyed by similar notions of "almost" minimizers, like $Q$-minimizers or $\omega$-minimizers (cf. Giusti [Gi], Dacorogna [D1]). This property is crucial for our methods and owes to the fact that deviation form minimality is viewed additively.

This paper is organized as follows. In Section 2 we present the core of our method, which consists of the construction of the approximations. Then, in Section 3 we employ them to establish our main results, the Maximum Principles. 


\section{Construction of the Approximations.}

Let us begin with some basics. In what follows, Sobolev functions will always be tacitly identified with their precise representatives. The Orthogonal group of $\mathbb{R}^{N}$ will be denoted by $O(N, \mathbb{R})$ and the Affine Orthogonal group of $\mathbb{R}^{N}$ by $A O(N, \mathbb{R})$.

Let $\xi \in \mathbb{S}^{N-1}$ be a vector in the unit sphere. $\xi$ defines a hyperplane $H_{\xi}:=$ $(\operatorname{span}[\xi])^{\perp} \subseteq \mathbb{R}^{N}$ and a reflection $R_{\xi} \in \mathrm{O}(\mathrm{N}, \mathbb{R})$ with respect to $H_{\xi}$, given by $R_{\xi} u:=u-2(u \cdot \xi) \xi$. Here, "." denotes inner product. If $u_{0} \in \mathbb{R}^{N}$, the affine reflection with respect to the hyperplane $H_{\xi}+u_{0}$ is given by $u \mapsto u-2\left(\left(u-u_{0}\right) \cdot \xi\right) \xi$ $=R_{\xi} u+2\left(u_{0} \cdot \xi\right) \xi$.

Let $\mathcal{C} \subseteq \mathbb{R}^{N}$ be compact and convex. We will write $\xi=\hat{u}$ for the outwards pointing normal to the supporting hyperplane $H_{\hat{u}}+u$ at $u$, but unless $\partial \mathcal{C}$ is differentiable, $u \mapsto \hat{u}$ may be multivalued and determines the normal cone. For any $u \in \partial \mathcal{C}, \hat{u}$ generates a reflection $w \mapsto R_{\hat{u}} w+2(u \cdot \hat{u}) \hat{u}$ in $\mathrm{AO}(\mathrm{N}, \mathbb{R})$ with respect to $H_{\hat{u}}+u$.

The following is the principal result of this section. It states that given any compact convex set, there exists a sequence of weak* approximations in $W_{l o c}^{1, \infty}\left(\mathbb{R}^{N}\right)^{N}$ of the projection on it generated by affine reflections merely.

Theorem 2.1 (Approximation of the projection onto convex sets by reflections). Let $\mathcal{C} \subseteq \mathbb{R}^{N}$ be a compact convex set with nonempty interior and $0 \in \operatorname{int}(\mathcal{C})$. Let also $\mathcal{P}^{\mathcal{C}}: \mathbb{R}^{N} \longrightarrow \mathcal{C}$ denote the projection on it.

Then, there exists a sequence of locally Lipschitz maps $\mathcal{F}_{m}^{\mathcal{C}}: \mathbb{R}^{N} \longrightarrow \mathbb{R}^{N}, m \in \mathbb{N}$, which satisfies:

(i) Each $\mathcal{F}_{m}^{\mathcal{C}}$ is piecewise equal to a composition of finitely many successive affine reflections. In particular, for a.e. $u \in \mathbb{R}^{N}$, there is an $R \in O(N, \mathbb{R})$ such that $D \mathcal{F}_{m}^{\mathcal{C}}(u)=R$.

(ii) Each $\mathcal{F}_{m}^{\mathcal{C}}$ equals the identity on $\mathcal{C}$ and maps the $m$-dilate of $\mathcal{C}$ into the $\left(1+\frac{1}{m}\right)$ dilate of $\mathcal{C}$ :

$$
\left\{\begin{array}{l}
\mathcal{F}_{m}^{\mathcal{C}}(E)=E, \quad E \subseteq \mathcal{C}, \\
\mathcal{F}_{m}^{\mathcal{C}}(m \mathcal{C}) \subseteq\left(1+\frac{1}{m}\right) \mathcal{C}
\end{array}\right.
$$

(iii) The sequence $\mathcal{F}_{m}^{\mathcal{C}}$ is a locally uniform approximation of the projection onto the convex set $\mathcal{C}$; moreover, we have $\mathcal{F}_{m}^{\mathcal{C}} \stackrel{*}{\longrightarrow} \mathcal{P}^{\mathcal{C}}$ weakly ${ }^{*}$ in $\left[W_{\text {loc }}^{1, \infty}\left(\mathbb{R}^{N}\right)\right]^{N}$, as $m \rightarrow \infty$.

The functions $\left\{\mathcal{F}_{m}^{\mathcal{C}} \mid m \in \mathbb{N}\right\}$ provided by Theorem 2.1 will be referred to as the Folding Maps of the convex set $\mathcal{C}$.

Example 2.2. An elementary example of Folding maps in the scalar case is given by the sequence defined inductively as $\mathcal{F}_{0}(u):=u \chi_{(0,1)}(u)+(2-u) \chi_{(1,2)}(u)$ and $\mathcal{F}_{m+1}:=\mathcal{F}_{m} \chi_{\left\{\mathcal{F}_{m}<2^{-(m+1)}\right\}}+\left(2^{-m}-\mathcal{F}_{m}\right) \chi_{\left\{\mathcal{F}_{m}>2^{-(m+1)}\right\}}$, in $W^{1, \infty}(0,2)$.

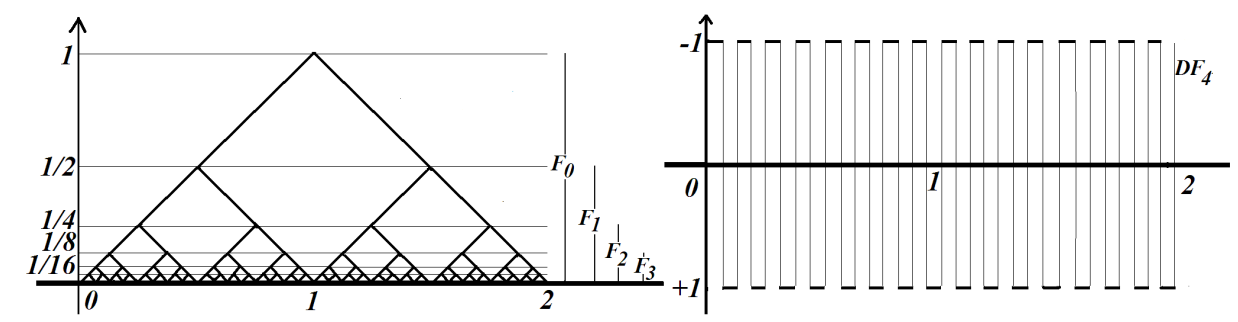


We have that $\left\|\mathcal{F}_{m}\right\|_{W^{1, \infty}(0,2)} \leq 1$ and since $\left\|\mathcal{F}_{m}\right\|_{L^{\infty}(0,2)} \leq 2^{-m-1}$, the weak* limit is zero. Thus $\mathcal{F}_{m} \stackrel{*}{\longrightarrow} 0$ in $W^{1, \infty}(0,2)$, which implies $\mathcal{F}_{m} \longrightarrow 0$ in $L^{\infty}(0,2)$. The sequence $\mathcal{F}_{m}$ approximates the projection $\mathcal{P}^{0}$ on $\{0\}$, which is the zero map.

Throughout the proof we will need to supplement convexity with an auxiliary notion which rectifies the lack of smoothness. It is eventually dropped by approximation.

Let $\mathcal{C}$ be a simplex, that is a compact set of the form $\bigcap\left\{H_{j}^{-}: 1 \leq j \leq K\right\}$ generated by closed halfspaces $H_{j}^{-}$which are determined by affine hyperplanes $H_{j}=\left(\operatorname{span}\left[\xi_{i}\right]\right)^{\perp}+u_{i}$. For each hyperplane $H_{i}, \xi_{i}$ is its unit normal vector pointing outwards of the halfspace $H_{i}^{-}$, while the hyperplane passes through $u_{i}$.

A simplex $\mathcal{C} \subseteq \mathbb{R}^{N}$ will be called obtuse, if its defining sides $H_{j}^{*}$ meet in obtuse angles $\geq \frac{\pi}{2}$, that is, when for all $i, j \in\{1, \ldots, K\}$ we have the implication

$$
H_{i}^{*} \cap H_{j}^{*} \neq \emptyset \Rightarrow 0 \leq \xi_{i} \cdot \xi_{j} \leq 1 .
$$

Proof of Theorem 2.1. Since $\mathcal{C}$ is fixed, we drop the superscript " $\mathcal{C}$ ". We shall first prove the existence of $\mathcal{F}_{m}$ for obtuse simplices satisfying (2.2) and then deduce the case of general convex sets by approximation. For, assume $\mathcal{C}=\bigcap\left\{H_{j}^{-}: 1 \leq j \leq K\right\}$ with $K \geq N+1$ and $H_{j}=u_{j}+\left(\operatorname{span}\left[\xi_{j}\right]\right)^{\perp}$ with (2.2) being satisfied by the sides $H_{i}^{*}$. We fix $m \in \mathbb{N}$. Then, $\mathcal{C} \subseteq\left(1+\frac{1}{m}\right) \mathcal{C}$ and since $\left(1+\frac{1}{m}\right) H_{j}=\left(1+\frac{1}{m}\right) u_{j}+$ $\left(\operatorname{span}\left[\xi_{j}\right]\right)^{\perp}$, we obtain

$$
\left(1+\frac{1}{m}\right) \mathcal{C}=\bigcap\left\{\left(1+\frac{1}{m}\right) H_{j}^{-}: 1 \leq j \leq K\right\} .
$$

We choose $\xi_{\min }$ on $\partial \mathcal{C}$ and $\xi_{\max }$ on $\bigcup\left\{H_{j}: 1 \leq j \leq K\right\}$ such that

$$
\begin{aligned}
& \left|\xi_{\min }\right|=\min _{1 \leq j \leq K}\left(\min _{u \in H_{j}}|u|\right), \\
& \left|\xi_{\max }\right|=\max _{1 \leq j \leq K}\left(\min _{u \in H_{j}}|u|\right) .
\end{aligned}
$$

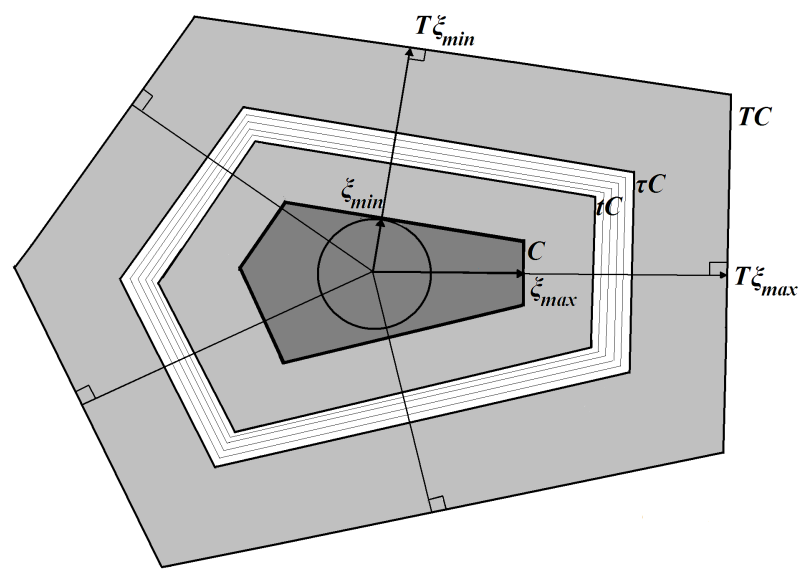

The points $\xi_{\min }$ and $\xi_{\max }$ always exist and are normal to $\bigcup\left\{H_{j}: 1 \leq j \leq K\right\}$, but possibly they are not unique or perhaps $\left|\xi_{\min }\right|=\left|\xi_{\max }\right|$. Let us set $T:=m$. The 
dilation $u \mapsto T u$ maps $\mathcal{C}$ onto $T \mathcal{C}$. We fix two numbers $t, \tau>0$ with $1<t<\tau \leq T$, chosen such that

$$
t=\frac{\tau\left|\xi_{\max }\right|+\left|\xi_{\min }\right|}{\left|\xi_{\min }\right|+\left|\xi_{\max }\right|}
$$

By (2.4) and (2.5), the minimum distance between the sides of $\partial \mathcal{C}$ and $\partial(t \mathcal{C})$ is realized along the $\xi_{\text {min }}$ direction, while the maximum distance between the sides of $\partial(\tau \mathcal{C})$ and $\partial(t \mathcal{C})$ along the $\xi_{\max }$ direction. Thus, (2.6) rearranged says $\left|(t-1) \xi_{\min }\right|=$ $\left|(\tau-t) \xi_{\max }\right|$, or

$$
\min _{1 \leq j \leq K}\left(\min _{u \in H_{j}}|t u-u|\right)=\max _{1 \leq j \leq K}\left(\min _{u \in H_{j}}|\tau u-t u|\right) .
$$

Let us fix $s \in[1, T]$ and $j \in\{1, \ldots, K\}$. The affine reflection $R_{j}^{s} \in \mathrm{AO}(\mathrm{N}, \mathbb{R})$ with respect to $s H_{j}$ is given by $R_{j}^{s}(u)=R_{\xi_{j}} u+2 s\left(u_{j} \cdot \xi_{j}\right) \xi_{j}, R_{\xi_{j}} \in O(N, \mathbb{R})$. For any $s \in[1, T]$ and $j \in\{1, \ldots, K\}$, we define

$$
\mathcal{F}_{j}^{s}(u):=\left\{\begin{array}{ll}
u, & u \in s H_{j}^{-} \\
R_{j}^{s}(u), & u \notin s H_{j}^{-}
\end{array}, \quad \mathcal{F}_{j}^{s}: \mathbb{R}^{N} \longrightarrow \mathbb{R}^{N},\right.
$$

and set

$$
\mathcal{F}^{s}:=\mathcal{F}_{K}^{s} \circ \cdots \circ \mathcal{F}_{1}^{s}, \quad \mathcal{F}^{s}: \mathbb{R}^{N} \longrightarrow \mathbb{R}^{N} .
$$

We obtain that $\mathcal{F}^{s}$ equals the identity on $s \mathcal{C}$ and for a.e. $u \in \mathbb{R}^{N}$, there is an $R \in O(N, \mathbb{R})$ such that $D \mathcal{F}^{s}(u)=R u$. Hence, $\left|D \mathcal{F}^{s}\right|=1$ a.e. on $\mathbb{R}^{N}$.

Claim 2.3. Assume that $t, \tau$ are in $[1, T]$ and they satisfy (2.6). Then, for all $j=1, \ldots, K$, we have

$$
\mathcal{F}_{j}^{t}\left(\tau \mathcal{C} \backslash \operatorname{int}\left(t H_{j}^{-}\right)\right) \subseteq \tau \mathcal{C} \cap\left(t H_{j}^{-} \backslash \operatorname{int}\left(H_{j}^{-}\right)\right)
$$

Proof of Claim 2.3. First note that by (2.6),

$$
\operatorname{dist}\left(\tau H_{j}^{-}, t H_{j}^{-}\right) \leq \operatorname{dist}\left(t H_{j}^{-}, H_{j}^{-}\right),
$$

for $j=1, . ., K$. Thus, $\mathcal{F}_{j}^{t}$ maps the zone $\tau H_{j}^{-} \backslash \operatorname{int}\left(t H_{j}^{-}\right)$into the zone $t H_{j}^{-} \backslash \operatorname{int}\left(H_{j}^{-}\right)$. Let us normalize up to an element of $\mathrm{AO}(\mathrm{N}, \mathbb{R})$ to $\xi_{j}=e_{N}, t H_{j}=\left\{u_{N}=0\right\}$. Let $v=\left(v^{\prime}, v_{N}\right)$ be in $\tau \mathcal{C} \backslash \operatorname{int}\left(t H_{j}^{-}\right)$. Then, we have $R_{j}^{t}(v)=\left(v^{\prime},-v_{N}\right)$ and $R_{j}^{t}(v)$ belongs to

$$
t H_{j}^{-} \backslash \operatorname{int}\left(H_{j}^{-}\right)=\left\{v \in \mathbb{R}^{N}:(1-t)\left|u_{j}\right| \leq v_{N} \leq 0\right\} .
$$

By obtuseness, it suffices to show that $R_{j}^{t}(v)$ is into

$$
\bigcap\left\{\tau H_{i}^{-}: i \neq j, H_{i}^{*} \cap H_{j}^{*} \neq \emptyset\right\},
$$

for all the neighbor sides $H_{i}^{*}$ of $H_{j}^{*}$. Let us fix such an $H_{i}^{*}$. Then, $\tau H_{i}=$ $\left(\operatorname{span}\left[\xi_{i}\right]\right)^{\perp}+\tau u_{i}$ and by a further translation we may assume $u_{i}=0$ (of course, the 
rest points $\left\{u_{l}: l \neq i\right\}$ relative to $H_{l}^{*}$ necessarily change, but this affects nothing).

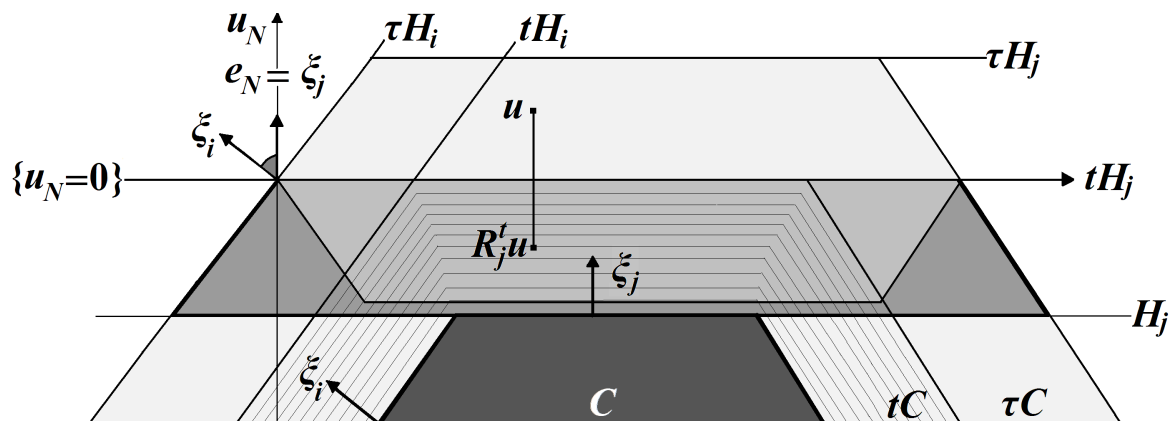

We obtain

$$
\begin{aligned}
\tau H_{i} & =\left\{z \in \mathbb{R}^{N}: z \cdot \xi_{i}=0\right\}, \\
\tau H_{i}^{-} & =\left\{z \in \mathbb{R}^{N}: z \cdot \xi_{i} \leq 0\right\} .
\end{aligned}
$$

Since $v \in \tau \mathcal{C}$ and

$$
\tau \mathcal{C}=\bigcap\left\{\tau H_{j}^{-}: 1 \leq j \leq K\right\} \subseteq \tau H_{i}^{-},
$$

we get that $v \cdot \xi_{i} \leq 0$ and by writing $v=\left(v^{\prime}, v_{N}\right)$ and $\xi_{i}=\left(\xi_{i}^{\prime}, \xi_{N}^{\prime}\right)$ and denoting inner products both in $\mathbb{R}^{N}$ and $\mathbb{R}^{N-1}$ by ".", we have

$$
\begin{aligned}
u^{\prime} \cdot \xi_{i}^{\prime} & =-v_{N}^{\prime} \xi_{i N}^{\prime}+v \cdot \xi_{i} \\
& =-\left(v \cdot e_{N}\right)\left(\xi_{i} \cdot e_{N}\right)+v \cdot \xi_{i} \\
& \leq-\left(v \cdot e_{N}\right)\left(\xi_{i} \cdot e_{N}\right) .
\end{aligned}
$$

Thus, utilizing that $v_{N} \geq 0$ and that obtuseness is equivalent to $0 \leq \xi_{i} \cdot e_{N} \leq 1$ for all such $i \neq j$, we obtain

$$
\begin{aligned}
R_{j}^{t}(v) \cdot \xi_{i} & =v^{\prime} \cdot \xi_{i}^{\prime}+\left(-v_{N}\right) \xi_{N}^{\prime} \\
& =v^{\prime} \cdot \xi_{i}^{\prime}-\left(v \cdot e_{N}\right)\left(\xi_{i} \cdot e_{N}\right) \\
& \leq-2\left(v \cdot e_{N}\right)\left(\xi_{i} \cdot e_{N}\right) \\
& \leq 0
\end{aligned}
$$

Consequently, if $v \in \tau \mathcal{C} \backslash \operatorname{int}\left(t H_{j}^{-}\right)$, we have $\mathcal{F}_{j}^{t}(v) \in \tau H_{i}^{-}$for all $i \neq j$ for which $H_{i}^{*} \cap H_{j}^{*} \neq \emptyset$.

Claim 2.4. Assume that $t, \tau$ belong to $[1, T]$ and that they satisfy (2.6). Then, we have

$$
\left\{\begin{array}{l}
(i) \mathcal{F}^{t}(\tau \mathcal{C} \backslash \operatorname{int}(t \mathcal{C})) \subseteq t \mathcal{C} \backslash \operatorname{int}(\mathcal{C}) \\
(i i) \mathcal{F}^{t}(t \mathcal{C})=t \mathcal{C}
\end{array}\right.
$$

Proof of Claim 2.4. Let $u \in \tau \mathcal{C} \backslash \operatorname{int}(t \mathcal{C})$ be a fixed point. We first show that $\mathcal{F}^{t}(u) \notin \operatorname{int}(\mathcal{C})$. Let $k \in\{1, \ldots, K\}$ be the first in order index for which $u \in \tau \mathcal{C} \backslash$ $\operatorname{int}\left(t H_{k}^{-}\right)$. By $(2.8)$, all $\mathcal{F}_{1}^{t}, \ldots, \mathcal{F}_{k-1}^{t}$ leave $u$ invariant, hence $\mathcal{F}^{t}(u)=\mathcal{F}_{K}^{t}\left(\ldots \mathcal{F}_{k}^{t}(u)\right)$. By Claim 2.3,

$$
\mathcal{F}_{k}^{t}(u) \in \tau \mathcal{C} \cap\left(t H_{k}^{-} \backslash \operatorname{int}\left(H_{k}^{-}\right)\right)
$$

and in particular $\mathcal{F}^{t}(u) \notin \operatorname{int}(\mathcal{C})$. Let $l \geq k+1$ be the next in order index for which

$$
\mathcal{F}_{k}^{t}(u) \in \tau \mathcal{C} \cap\left(t H_{k}^{-} \backslash \operatorname{int}\left(H_{k}^{-}\right)\right) \backslash \operatorname{int}\left(t H_{l}^{-}\right) .
$$


For all the intermediate indices $k+1 \leq i<l, \mathcal{F}_{i}^{t}$ leaves $\mathcal{F}_{k}^{t}(u)$ invariant and hence

$$
\mathcal{F}_{l}^{t}\left(\mathcal{F}_{l-1}^{t} \ldots \mathcal{F}_{i}^{t} \ldots\left(\mathcal{F}_{k}^{t}(u)\right)\right)=\mathcal{F}_{l}^{t}\left(\mathcal{F}_{k}^{t}(u)\right)
$$

By Claim 2.3, we have

$$
\mathcal{F}_{l}^{t}\left(\mathcal{F}_{k}^{t}(u)\right) \in t H_{l}^{-} \backslash \operatorname{int}\left(H_{l}^{-}\right)
$$

and thus $\mathcal{F}_{l}^{t}\left(\mathcal{F}_{k}^{t}(u)\right) \notin \operatorname{int}(\mathcal{C})$. In view on $(2.9)$, by continuing till $K$ we obtain $\mathcal{F}^{t}(u) \notin \operatorname{int}(\mathcal{C})$.

We now show that $\mathcal{F}^{t}(u) \in \operatorname{int}(t \mathcal{C})$. Let again $k \in\{1, \ldots, K\}$ be the first in order index for which $u \in \tau \mathcal{C} \backslash t H_{k}^{-}$. Then, $\mathcal{F}^{t}(u)=\mathcal{F}_{K}^{t}\left(\ldots \mathcal{F}_{k}^{t}(u)\right)$. Let $H_{l}^{*}, l \geq k+1$, be the first in order neighbor side of $H_{k}^{*}$. By Claim 2.3, we have

$$
\mathcal{F}_{k}^{t}(u) \in \tau \mathcal{C} \cap\left(t H_{k}^{-} \backslash \operatorname{int}\left(H_{k}^{-}\right)\right) .
$$

If $\mathcal{F}_{k}^{t}(u) \in t H_{l}^{-} \cap \tau \mathcal{C}$, we proceed to the next neighbor side. Otherwise, if $\mathcal{F}_{k}^{t}(u) \in$ $\tau \mathcal{C} \backslash \operatorname{int}\left(t H_{l}^{-}\right)$, the exact same argument in the proof of Claim 2.3 with $t H_{k}$ in the place of $t H_{j}$ and $t H_{l}$ in the place of $\tau H_{i}$ shows that

$$
\mathcal{F}_{l}^{t}\left(\mathcal{F}_{k}^{t}(u)\right) \in\left(t H_{k}^{-} \backslash \operatorname{int}\left(H_{k}^{-}\right)\right) \cap\left(t H_{l}^{-} \backslash \operatorname{int}\left(H_{l}^{-}\right)\right) .
$$

In view of (2.9), continuing till $K$ we obtain

$$
\mathcal{F}^{t}(u) \in \bigcap\left\{t H_{j}^{-} \backslash \operatorname{int}\left(H_{j}^{-}\right): 1 \leq j \leq K\right\}
$$

and hence $\mathcal{F}^{t}(u) \in t \mathcal{C}$, as desired.

(ii) follows directly from (2.8) and (2.9).

Now we iterate Claim 2.4. We define inductively:

$$
\left\{\begin{aligned}
t_{0} & :=T \\
t_{k+1} & :=\frac{t_{k}\left|\xi_{\max }\right|+\left|\xi_{\min }\right|}{\left|\xi_{\min }\right|+\left|\xi_{\max }\right|} .
\end{aligned}\right.
$$

Claim 2.5. The sequence $\left(t_{k}\right)_{1}^{\infty}$ defined by (2.26) is strictly decreasing to $1^{+}$as $k \rightarrow \infty$, and

$$
t_{k}=\left(\frac{\left|\xi_{\max }\right|}{\left|\xi_{\max }\right|+\left|\xi_{\min }\right|}\right)^{k} T+\left(\frac{\left|\xi_{\min }\right|}{\left|\xi_{\max }\right|+\left|\xi_{\min }\right|}\right) \sum_{j=0}^{k-1}\left(\frac{\left|\xi_{\max }\right|}{\left|\xi_{\max }\right|+\left|\xi_{\min }\right|}\right)^{j}
$$

for all $k \in \mathbb{N}$.

Proof of Claim 2.5. Follows by induction and the geometric series.

For $m \in \mathbb{N}$, we choose $k(m) \in \mathbb{N}$ such that $t_{k(m)}<1+\frac{1}{m}$. Then, $t_{k(m)} \mathcal{C} \subseteq\left(1+\frac{1}{m}\right) \mathcal{C}$. We define:

$$
\mathcal{F}_{m}^{\mathcal{C}}:=\mathcal{F}^{t_{k(m)}} \circ \cdots \circ \mathcal{F}^{t_{1}}
$$

Claim 2.6. The Folding Map $\mathcal{F}_{m}^{\mathcal{C}}: \mathbb{R}^{N} \longrightarrow \mathbb{R}^{N}$ given by (2.8), (2.9), (2.28) is a locally Lipschitz map in $\left[W_{\text {loc }}^{1, \infty}\left(\mathbb{R}^{N}\right)\right]^{N}$, piecewise equal to compositions of affine reflections and satisfies (2.1) for all $m \in \mathbb{N}$.

Proof of Claim 2.6. By Claims 2.4, 2.5, for all $k=1,2, \ldots$ we have

$$
\left\{\begin{array}{l}
\mathcal{F}^{t_{k}}\left(t_{k-1} \mathcal{C} \backslash \operatorname{int}\left(t_{k} \mathcal{C}\right)\right) \subseteq t_{k} \mathcal{C} \backslash \operatorname{int}(\mathcal{C}) \\
\mathcal{F}^{t_{k}}\left(t_{k} \mathcal{C}\right)=t_{k} \mathcal{C}
\end{array}\right.
$$


By Definitions (2.8), (2.9), (2.28), we readily have that $\mathcal{F}_{m}^{\mathcal{C}}$ leaves $\mathcal{C}$ invariant; thus, $\mathcal{F}_{m}^{\mathcal{C}}(E)=E$ for all $E \subseteq \mathcal{C}$. The rest properties of $\mathcal{F}_{m}^{\mathcal{C}}$ also follow by construction, so it suffices to establish (2.1). For, by employing that $m=T=t_{0}$, we obtain

$$
\begin{aligned}
\mathcal{F}_{m}^{\mathcal{C}}(m \mathcal{C}) & \subseteq \mathcal{F}^{t_{k(m)}}\left(\ldots \mathcal{F}^{t_{1}}\left(t_{0} \mathcal{C}\right)\right) \\
& \subseteq \mathcal{F}^{t_{k(m)}}\left(\ldots \mathcal{F}^{t_{2}}\left(t_{1} \mathcal{C}\right)\right) \\
& \subseteq \mathcal{F}^{t_{k(m)}}\left(\ldots \mathcal{F}^{t_{3}}\left(t_{2} \mathcal{C}\right)\right) \\
& \subseteq \ldots \\
& \subseteq t_{k(m)} \mathcal{C} \\
& \subseteq\left(1+\frac{1}{m}\right) \mathcal{C} .
\end{aligned}
$$

Now, since $T \mathcal{C} \backslash \operatorname{int}(\mathcal{C})=t_{0} \mathcal{C} \backslash \operatorname{int}(\mathcal{C})$, by utilizing $(2.8),(2.9),(2.28)$ we have

$$
\begin{aligned}
\mathcal{F}^{t_{1}}(m \mathcal{C} \backslash \mathcal{C}) \subseteq & \mathcal{F}^{t_{1}}\left(t_{0} \mathcal{C} \backslash \operatorname{int}(\mathcal{C})\right) \\
= & \mathcal{F}^{t_{1}}\left(\left[\left(t_{0} \mathcal{C} \backslash \operatorname{int}(\mathcal{C})\right) \cap \operatorname{int}\left(t_{1} \mathcal{C}\right)\right]\right. \\
& \left.\cup\left[\left(t_{0} \mathcal{C} \backslash \operatorname{int}(\mathcal{C})\right) \backslash \operatorname{int}\left(t_{1} \mathcal{C}\right)\right]\right) \\
\subseteq & \left(t_{1} \mathcal{C} \backslash \operatorname{int}(\mathcal{C})\right) \cup \mathcal{F}^{t_{1}}\left(t_{0} \mathcal{C} \backslash \operatorname{int}\left(t_{1} \mathcal{C}\right)\right) \\
\subseteq & \left(t_{1} \mathcal{C} \backslash \operatorname{int}(\mathcal{C})\right) \cup\left(t_{1} \mathcal{C} \backslash \operatorname{int}(\mathcal{C})\right) \\
= & t_{1} \mathcal{C} \backslash \operatorname{int}(\mathcal{C}), \\
\mathcal{F}^{t_{2}}\left(\mathcal{F}^{t_{1}}(m \mathcal{C} \backslash \mathcal{C})\right) \subseteq & \mathcal{F}^{t_{2}}\left(t_{1} \mathcal{C} \backslash \operatorname{int}(\mathcal{C})\right) \\
= & \mathcal{F}^{t_{2}}\left(\left[\left(t_{1} \mathcal{C} \backslash \operatorname{int}(\mathcal{C})\right) \cap \operatorname{int}\left(t_{2} \mathcal{C}\right)\right]\right. \\
& \left.\cup\left[\left(t_{1} \mathcal{C} \backslash \operatorname{int}(\mathcal{C})\right) \backslash \operatorname{int}\left(t_{2} \mathcal{C}\right)\right]\right) \\
\subseteq & \left(t_{2} \mathcal{C} \backslash \operatorname{int}(\mathcal{C})\right) \cup \mathcal{F}^{t_{2}}\left(t_{1} \mathcal{C} \backslash \operatorname{int}\left(t_{2} \mathcal{C}\right)\right) \\
\subseteq & \left(t_{2} \mathcal{C} \backslash \operatorname{int}(\mathcal{C})\right) \cup\left(t_{2} \mathcal{C} \backslash \operatorname{int}(\mathcal{C})\right) \\
= & t_{2} \mathcal{C} \backslash \operatorname{int}(\mathcal{C}), \\
\vdots & \\
\mathcal{F}_{m}^{\mathcal{C}}(m \mathcal{C} \backslash \mathcal{C})= & \mathcal{F}^{t_{k(m)}}\left(\ldots \mathcal{F}^{t_{1}}(m \mathcal{C} \backslash \operatorname{int}(\mathcal{C}))\right) \\
\subseteq & \ldots \\
\subseteq & t_{k(m)} \mathcal{C} \backslash \operatorname{int}(\mathcal{C}) \\
\subseteq & \left(1+\frac{1}{m}\right) \mathcal{C} \backslash \operatorname{int}(\mathcal{C}) \\
\subseteq & \left(1+\frac{1}{m}\right) \mathcal{C} . \\
&
\end{aligned}
$$

Claim 2.6 has been established.

So far, we have established $(i)$ and $(i i)$ of Theorem 2.1 under the assumption that $\mathcal{C}$ is an obtuse simplex. Let us now drop this assumption.

Claim 2.7. Statements $(i)$ and $(i i)$ of Theorem 2.1 hold for a general compact convex set $\mathcal{C}$ with $0 \in \operatorname{int}(\mathcal{C})$. 
Proof of Claim 2.7. Let $\left(\mathcal{C}_{k}\right)_{k=1}^{\infty}$ be a decreasing sequence of $C^{1}$ compact convex sets which approximates $\mathcal{C}$ from the outside:

$$
\mathcal{C}_{1} \supseteq \mathcal{C}_{2} \supseteq \ldots \supseteq \mathcal{C}_{k} \supseteq \ldots \supseteq \mathcal{C} .
$$

Fix $k \in \mathbb{N}$. Since $\mathcal{C}_{k}$ is $C^{1}$, if $p, q \in \partial \mathcal{C}$ and $\hat{p}, \hat{q}$ are the outwards pointing normal vectors at $p, q$, then regularity and compactness imply the existence of a continuous increasing modulus of continuity $\omega \in C^{0}[0, \infty)$ with $\omega(0)=0$ such that

$$
|\hat{p}-\hat{q}| \leq \omega(|p-q|)
$$

By employing estimate (2.35), we obtain

$$
\begin{aligned}
|1-\cos (\operatorname{Angl}(\hat{p}, \hat{q}))| & =\left|\frac{1}{2}\left(|\hat{p}|^{2}+|\hat{q}|^{2}\right)-\right| \hat{p}|| \hat{q}|\cos (\operatorname{Angl}(\hat{p}, \hat{q}))| \\
& =\left.\frac{1}{2}|| \hat{p}\right|^{2}+|\hat{q}|^{2}-2(\hat{p} \cdot \hat{q}) \mid \\
& =\frac{1}{2}|\hat{p}-\hat{q}|^{2} \\
& \leq \frac{1}{2} \omega^{2}(|p-q|) .
\end{aligned}
$$

Hence, by (2.36), if $p$ is close to $q$, the tangent hyperplanes at these points meet at obtuse angles. By (2.36), each smooth convex set $\mathcal{C}_{k}$ can be approximated from the outside by a decreasing sequence $\left(\mathcal{C}_{k, l}\right)_{l=1}^{\infty}$ of obtuse simplices, generated by tangent hyperplanes:

$$
\mathcal{C}_{k, 1} \supseteq \mathcal{C}_{k, 2} \supseteq \ldots \supseteq \mathcal{C}_{k, l} \supseteq \ldots \supseteq \mathcal{C}_{k}
$$

Without harming generality, we may assume that $l$ denotes the number of sides of the simplex $\mathcal{C}_{k, l}$. If $m \in \mathbb{N}$, let us define the Folding Map $\mathcal{F}_{m}^{\mathcal{C}}$ of $\mathcal{C}$ as the $l(m)$ Folding Map referring to the obtuse simplex $\mathcal{C}_{k(m), l(m)}$ for some $k(m), l(m) \in \mathbb{N}$ sufficiently large:

$$
\mathcal{F}_{m}^{\mathcal{C}}:=\mathcal{F}_{l(m)}^{\mathcal{C}_{k(m), l(m)}}
$$

By the previous analysis, such numbers $k(m), l(m)$ exist and $\mathcal{F}_{m}^{\mathcal{C}}: \mathbb{R}^{N} \longrightarrow \mathbb{R}^{N}$ has all the desired properties of $(i)$ and $(i i)$.

Let us conclude Theorem 2.1 by establishing (iii).

Claim 2.8. There exists subsequences $(k(m))_{m=1}^{\infty},(l(m))_{m=1}^{\infty}$ such that the Folding map of $\mathcal{C}$ given by (2.8), (2.9), (2.28) and (2.38) satisfies $\mathcal{F}_{m}^{\mathcal{C}} \stackrel{*}{\longrightarrow} \mathcal{P}^{\mathcal{C}}$ weakly ${ }^{*}$ in $\left[W_{\text {loc }}^{1, \infty}\left(\mathbb{R}^{N}\right)\right]^{N}$, along a subsequence as $m \rightarrow \infty$.

Proof of Claim 2.8. By Claims $2.3-2.7$, for any $m \in \mathbb{N}$ and any $j \leq m$, we have

$$
\begin{aligned}
\mathcal{F}_{m}^{\mathcal{C}}(j \mathcal{C} \backslash \mathcal{C}) & \subseteq\left(1+\frac{1}{m}\right) \mathcal{C} \backslash \operatorname{int}(\mathcal{C}), \\
\mathcal{F}_{m}^{\mathcal{C}}(E) & =E, \quad E \subseteq \mathcal{C} \\
\left|D \mathcal{F}_{m}^{\mathcal{C}}\right| & =1, \text { a.e. on } \mathbb{R}^{N} .
\end{aligned}
$$

Hence, by (2.39) - (2.41) we obtain the bounds

$$
\begin{aligned}
\left\|\mathcal{F}_{m}^{\mathcal{C}}\right\|_{L^{\infty}(j \mathcal{C})} & \leq 2 \operatorname{diam}(\mathcal{C}) \\
\left\|D \mathcal{F}_{m}^{\mathcal{C}}\right\|_{L^{\infty}\left(\mathbb{R}^{N}\right)} & \leq 1
\end{aligned}
$$


for all $j \leq m$ and $m \in \mathbb{N}$. By (2.42), (2.43), weak* compactness of the local space $\left[W_{\text {loc }}^{1, \infty}\left(\mathbb{R}^{N}\right)\right]^{N}$ and local compactness of the imbedding $W_{\text {loc }}^{1, \infty}\left(\mathbb{R}^{N}\right) \subset \subset L_{\text {loc }}^{\infty}\left(\mathbb{R}^{N}\right)$, we can extract a subsequence (denoted again by $\mathcal{F}_{m}^{\mathcal{C}}$ ) such that, for some $\mathcal{F}^{\mathcal{C}}$ in $\left[W_{\text {loc }}^{1, \infty}\left(\mathbb{R}^{N}\right)\right]^{N}$

$$
\begin{array}{rl}
\mathcal{F}_{m}^{\mathcal{C}} & \longrightarrow \mathcal{F}^{\mathcal{C}}, \text { in }\left[L_{\mathrm{loc}}^{\infty}\left(\mathbb{R}^{N}\right)\right]^{N}, \\
D \mathcal{F}_{m}^{\mathcal{C}} & * D \mathcal{F}^{\mathcal{C}}, \text { in }\left[L_{\mathrm{loc}}^{\infty}\left(\mathbb{R}^{N}\right)\right]^{N \times N},
\end{array}
$$

as $m \rightarrow \infty$. By passing to the locally uniform limit to (2.39) and (2.40) and then letting $j \rightarrow \infty$, we obtain

$$
\begin{aligned}
& \mathcal{F}^{\mathcal{C}}\left(\mathbb{R}^{N} \backslash \mathcal{C}\right) \subseteq \partial \mathcal{C}, \\
& \mathcal{F}^{\mathcal{C}}(E)=E, \quad E \subseteq \mathcal{C} .
\end{aligned}
$$

Let us complete Theorem 2.1 by establishing that for an appropriate choice of $k(m)$ and $l(m)$, the limit $\mathcal{F}^{\mathcal{C}}$ coincides with the projection $\mathcal{P}^{\mathcal{C}}$ on $\mathcal{C}$. Fix $j, m \in \mathbb{N}$ and $\varepsilon>0$. Let $\mathcal{P}^{\mathcal{C}_{k(m)}}$ be the projection on $\mathcal{C}_{k(m)}$. Consider the estimate

$$
\begin{aligned}
\left\|\mathcal{F}^{\mathcal{C}}-\mathcal{P}^{\mathcal{C}}\right\|_{L^{\infty}(j \mathcal{C})} \leq & \left\|\mathcal{F}^{\mathcal{C}}-\mathcal{F}_{m}^{\mathcal{C}}\right\|_{L^{\infty}(j \mathcal{C})}+\left\|\mathcal{F}_{m}^{\mathcal{C}}-\mathcal{P}^{\mathcal{C}}{ }_{k(m)}\right\|_{L^{\infty}(j \mathcal{C})} \\
& +\left\|\mathcal{P}^{\mathcal{C}_{k(m)}}-\mathcal{P}^{\mathcal{C}}\right\|_{L^{\infty}(j \mathcal{C})} .
\end{aligned}
$$

By the estimate

$$
\begin{aligned}
& \left\|\mathcal{P}^{\mathcal{C}_{k(m)}}-\mathcal{P}^{\mathcal{C}}\right\|_{L^{\infty}(j \mathcal{C})} \leq \max \left\{\left|p-p_{m}\right|: p \in \partial \mathcal{C}, p_{m} \in \mathcal{C}_{k(m)},\right. \\
& \left.\left[p, p_{m}\right] \subseteq \mathcal{C}_{k(m)} \backslash \operatorname{int}(\mathcal{C})\right\},
\end{aligned}
$$

if $k(m)$ is chosen large enough, then

$$
\left\|\mathcal{P}^{\mathcal{C}_{k(m)}}-\mathcal{P}^{\mathcal{C}}\right\|_{L^{\infty}(j \mathcal{C})} \leq \varepsilon
$$

Consider now the sequence $\left(\mathcal{F}_{l}^{\mathcal{C}_{k(m), l}}\right)_{l=1}^{\infty}$ of Folding maps generated by the obtuse simplices $\mathcal{C}_{k(m), l}$ which approximate $\mathcal{C}_{k(m)}$. The sequence $\left(\mathcal{F}_{l}^{\mathcal{C}_{k(m), l}}\right)_{l=1}^{\infty}$ satisfies estimates $(2.42),(2.43)$ and by compactness has a locally uniform limit $\mathcal{F}^{\mathcal{C}_{k(m)}} \in$ $\left[L_{l o c}^{\infty}\left(\mathbb{R}^{N}\right)\right]^{N}:$

$$
\mathcal{F}_{l}^{\mathcal{C}_{k(m), l}} \longrightarrow \mathcal{F}^{\mathcal{C}_{k(m)}}
$$

in $\left[L_{l o c}^{\infty}\left(\mathbb{R}^{N}\right)\right]^{N}$ as $l \rightarrow \infty$, along a subsequence. We claim that $\mathcal{F}^{\mathcal{C}_{k(m)}}=\mathcal{P}^{\mathcal{C}_{k(m)}}$. For, observe that since the boundary of $\mathcal{C}_{k(m), l}$ consists of tangent hyperplanes to the $C^{1}$ convex set $\mathcal{C}_{k(m)}$ and $l$ is the number of sides, there exists a dense sequence $\left(u_{i}\right)_{1}^{\infty} \subseteq \partial \mathcal{C}_{k(m)}$, a respective sequence of outwards pointing normal vectors $\left(\hat{u}_{i}\right)_{1}^{\infty}$ and a strictly increasing function $\sigma \in C^{0}[0, \infty)$ with $\sigma(0)=0$ such that

$$
\mathcal{F}_{l}^{\mathcal{C}_{k(m), l}}\left(u_{i}+t \hat{u}_{i}\right)=u_{i}+\sigma\left(2^{-l}\right) \hat{u}_{i}
$$

for all $i \leq l$ and $t \in[0, \sigma(l)]$. Since the set of half-lines

$$
D:=\bigcup_{i=1}^{\infty}\left(u_{i}+\left\{t \hat{u}_{i}: t \geq 0\right\}\right)
$$


is dense in $\mathbb{R}^{N} \backslash \mathcal{C}$, by passing to the limit to $(2.52)$ as $l \rightarrow \infty$, we obtain

$$
\begin{aligned}
\mathcal{F}^{\mathcal{C}_{k(m)}}\left(u_{i}+t \hat{u}_{i}\right) & =u_{i} \\
& =\mathcal{P}^{\mathcal{C}_{k(m)}}\left(u_{i}+t \hat{u}_{i}\right),
\end{aligned}
$$

for all $i \in \mathbb{N}$ and all $t \in[0,+\infty)$. Consequently, $\mathcal{F}^{\mathcal{C}_{k(m)}}=\mathcal{P}^{\mathcal{C}_{k(m)}}$ on the dense set $D$; equality on $\mathbb{R}^{N} \backslash \mathcal{C}$ follows by continuity. Thus, by (2.51) we can choose an $l(m) \in \mathbb{N}$ sufficiently large to get

$$
\left\|\mathcal{F}_{l(m), l(m)}^{\mathcal{C}_{k(m)}}-\mathcal{P}^{\mathcal{C}_{k(m)}}\right\|_{L^{\infty}(j \mathcal{C})} \leq \varepsilon
$$

Finally, by (2.44) and (2.38), we can increase $l(m)$ further to assure

$$
\| \mathcal{F}_{l(m)}^{\mathcal{C}_{k(m), l(m)}-\mathcal{F}^{\mathcal{C}} \|_{L^{\infty}(j \mathcal{C})} \leq \varepsilon}
$$

By putting estimates (2.48), (2.50), (2.55) and (2.56) together, the desired conclusion $\mathcal{F}^{\mathcal{C}} \equiv \mathcal{P}^{\mathcal{C}}$ follows by the arbitrariness of $\varepsilon>0$ and $j \in \mathbb{N}$.

The proof of Theorem 2.1 is now complete.

\section{The Maximum Principles.}

In this section we present our main results, the Maximum Principles for approximate minimizers of nonconvex functionals, in the sense of the Convex Hull Property. They are obtained by employing the approximating Folding Maps of Theorem 2.1 to generate appropriate energy comparison functions which compensate the lack of weak Lower Semi-Continuity. We shall refer to them as Folding Principles, since they are obtained by employing the Folding Maps of Section 2.

Before proceeding to our main results, we first need to formulate the notions of " $u(\Omega)$ " and " $u(\partial \Omega)$ " in a weak sense, meaningful for merely measurable functions. Let $u: \Omega \subseteq \mathbb{R}^{n} \longrightarrow \mathbb{R}^{N}$ be a measurable function defined on the open set $\Omega$ and let $A, K \subseteq \bar{\Omega}$ be measurable as well. Let also $|-|$ denote the Lebesgue measure in any dimension. If $|A|>0$, the essential range $u(A)$ is the closed set

$$
u(A):=\left\{\eta \in \mathbb{R}^{N}|\underset{x \in A}{\operatorname{ess} \inf }| u(x)-\eta \mid=0\right\} .
$$

If $|K|=0$, (3.1) is not directly meaningful for $K$. We therefore define

$$
u(K):=\bigcap\{u(A)|A \supseteq K,| A \mid>0\} .
$$

Finally, if $S \subseteq \mathbb{R}^{N}$, we denote the open $\varepsilon$-neighborhood of $S$ by

$$
S^{\varepsilon}:=\left\{\eta \in \mathbb{R}^{N}|| \eta-s \mid<\varepsilon, s \in S\right\} .
$$

3.1. The case of integrand with no direct dependence on the function. We consider first the simpler case of the functional

$$
E(u, \Omega)=\int_{\Omega} \mathscr{L}(x, D u(x)) d x .
$$

Hypotheses on the functional (3.4). We shall need to impose the following assumptions: let $\Omega \subseteq \mathbb{R}^{n}$ be open and $\mathscr{L}: \Omega \times \mathbb{R}^{N \times n} \longrightarrow \mathbb{R}$ a Carathéodory function such that 
(1) $\mathscr{L}\left(x,_{-}\right)$is almost invariant under the Orthogonal Group: there exists $a \in$ $L^{1}(\Omega)$ such that for a.e. $x \in \Omega$, all $O \in O(N, \mathbb{R})$ and all $P \in \mathbb{R}^{N \times n}$, we have

$$
|\mathscr{L}(x, P)-\mathscr{L}(x, O P)| \leq a(x) .
$$

(2) $\mathscr{L}\left(x,{ }_{-}\right)$is of $q$-growth: there exist $C>0, q \geq 1$ and $b \in L^{1}(\Omega)$ such that

$$
-b(x) \leq \mathscr{L}(x, P) \leq C|P|^{q}+b(x)
$$

Remark 3.1. Assumption (2) is rather standard. Assumption (1) is always satisfied if $\mathscr{L}\left(x,,_{-}\right)$is invariant under $O(N, \mathbb{R})$, in which case $a \equiv 0$. In particular, this always holds in the frequent case where $\mathscr{L}$ depends on $P$ through its modulus: $\mathscr{L}=\mathscr{L}(x,|P|)$.

In the scalar case of $N=1$, assumption (1) requires

$$
|\mathscr{L}(x, P)-\mathscr{L}(x,-P)| \leq a(x),
$$

which means that $\mathscr{L}\left(x,{ }_{-}\right)$is almost even. Even in the scalar case, evenness which occurs for $a \equiv 0$ and reads $\mathscr{L}(x, P)=\mathscr{L}(x,-P)$, is an assumption much weaker than requiring to be radial, i.e. $\mathscr{L}=\mathscr{L}(x,|P|)$.

We note that the form $\mathscr{L}=\mathscr{L}(x,|P|)$ of the integrand coupled by monotonicity of the function $t \mapsto \mathscr{L}(x, t)$ is a standing assumption in the literature for the derivation of the Convex Hull Property. In this work we weaken it substantially and in particular we bypass quasiconvexity.

Theorem 3.2 (Folding Principle I). Let $\Omega \subseteq \mathbb{R}^{n}$ be open and $\mathscr{L}: \Omega \times \mathbb{R}^{N \times n} \longrightarrow \mathbb{R}$ a Carathédory function satisfying assumptions (1) and (2). Then, for any $\alpha>0$ and $g \in\left[W^{1, q}(\Omega) \cap L^{\infty}(\Omega)\right]^{N}$ there exists an $\left(\alpha+\|a\|_{L^{1}(\Omega)}\right)$-minimizer $u$ of the functional (3.4) in $\left[W_{g}^{1, q}(\Omega)\right]^{N}$ which satisfies

$$
u(\Omega) \subseteq \overline{c o}\left(u(\partial \Omega)^{\alpha}\right)
$$

By Theorem 3.2 we readily obtain the following

Corollary 3.3. In the setting of Theorem 3.2, if moreover $\mathscr{L}$ is invariant under $O(N, \mathbb{R})$, that is $a \equiv 0$ in assumption (1), then there exists a minimizing family $\left\{u_{\alpha}\right\}_{\alpha>0}$ of problem (1.3) which asymptotically satisfies the Convex Hull Property as $\alpha \rightarrow 0$ :

$$
u_{\alpha}(\Omega) \subseteq \overline{c o}\left(u_{\alpha}(\partial \Omega)^{\alpha}\right)
$$

Remark 3.4. By employing standard results (cf. for example Dacorogna [D1], [D3]), (3.7) is inherited by the relaxed solution of (1.3) and by minimizing solutions of the Euler-Lagrange system of PDEs, if they exist.

Remark 3.5. In the absence of weak Lower Semi-Continuity, (3.6) and (3.7) is all we can assert, since we can not pass to the limit as $\alpha \rightarrow 0$ to sharpen them. Moreover, if $\mathscr{L}$ is not invariant under the Orthogonal Group, then the Convex Hull Property is generally satisfied by an approximate minimizer at a higher energy level, increased by the amount " $\|a\|_{L^{1}(\Omega)}$ " of deviation of $\mathscr{L}$ from invariance.

Proof of Theorem 3.2. Fix $\alpha>0$. Under the lower $L^{1}$-bound of assumption (2), minimizing sequences of problem (1.3) are equivalent to families of approximate 
minimizers as $\alpha \rightarrow 0$. Hence, we can choose an $\frac{\alpha}{2}$-minimizer $v \in\left[W_{g}^{1, q}(\Omega)\right]^{N}$ of (3.4). We consider the $\frac{\alpha}{2}$-neighborhood of $u(\partial \Omega)$ and set

$$
\mathcal{C}:=\overline{\operatorname{co}}\left(u(\partial \Omega)^{\alpha / 2}\right)
$$

Since $u-g \in\left[W_{0}^{1, q}(\Omega)\right]^{N}$ and $g \in\left[L^{\infty}(\Omega)\right]^{N}$, by (3.1) and (3.2), $\mathcal{C}$ is a compact convex of $\mathbb{R}^{N}$ with nonempty interior. By a translation, we may assume that $0 \in \operatorname{int}(\mathcal{C})$. Let $\mathcal{F}_{m}, m \in \mathbb{N}$, be the Folding Maps of Theorem 2.1 relative to $\mathcal{C}$. Let also $v^{m}$ be the truncate of $v$ whose range is contained in the largest ball centered at zero inside the $m$-dilate $m \mathcal{C}$ of $\mathcal{C}$ :

$$
\begin{gathered}
v^{m}:=v \chi_{\{|v| \leq R(m)\}}+\frac{v}{|v|} \chi_{\{|v|>R(m)\}}, \\
R(m):=\max \{R \in \mathbb{N} \mid v(\{|v| \leq R\}) \subseteq m \mathcal{C}\} .
\end{gathered}
$$

Then, $v^{m} \rightarrow v$ in $\left[W^{1, q}(\Omega)\right]^{N}$ and $D v^{m} \rightarrow D v$ a.e. on $\Omega$ as well, along a certain subsequence as $m \rightarrow \infty$. Moreover, by Theorem 2.1, the transformation $\mathcal{F}_{m} \circ v^{m}$ is well-defined and contained in $\left[W^{1, q}(\Omega)\right]^{N}$. Moreover, for $m=m(\alpha)$ large, in view of (2.1) it satisfies

$$
\begin{aligned}
\left(\mathcal{F}_{m} \circ v^{m}\right)(\Omega) & \subseteq\left(1+\frac{1}{m}\right) \mathcal{C} \\
& \subseteq \mathcal{C}^{\alpha / 2} \\
& =\overline{\mathrm{CO}}\left(u(\partial \Omega)^{\alpha / 2}\right)^{\alpha / 2} \\
& =\overline{\mathrm{CO}}\left(u(\partial \Omega)^{\alpha}\right)
\end{aligned}
$$

By (2.1), $\mathcal{F}_{m}$ leaves $\mathcal{C}$ invariant; hence, by (3.1), (3.2) and (3.8) we obtain

$$
v(\partial \Omega)=\left(\mathcal{F}_{m} \circ v^{m}\right)(\partial \Omega) .
$$

Thus, $\mathcal{F}_{m} \circ v^{m} \in\left[W_{g}^{1, q}(\Omega)\right]^{N}$ and by (3.11) and (3.12) we obtain

$$
\left(\mathcal{F}_{m} \circ v^{m}\right)(\Omega) \subseteq \overline{\mathrm{co}}\left(\mathcal{F}_{m} \circ v^{m}\right)(\partial \Omega) .
$$

By assumption (2) and since $\left|D v^{m}\right| \leq 2|D v|$ for $m$ large, we have the bound

$$
\left|\mathscr{L}\left(x, D v^{m}(x)\right)\right| \leq 2^{q} C|D v(x)|^{q}+b(x),
$$

valid for a.e. $x \in \Omega$. Hence, since $D v^{m} \rightarrow D v$ a.e. as $m \rightarrow \infty$, the Dominated Convergence theorem implies

$$
\int_{\Omega} \mathscr{L}\left(x, D v^{m}(x)\right) d x \longrightarrow \int_{\Omega} \mathscr{L}(x, D v(x)) d x
$$


as $m \rightarrow \infty$. By Theorem 2.1, for a.e. $x \in \Omega$, we have that $D \mathcal{F}_{m}\left(v^{m}(x)\right) \in O(N, \mathbb{R})$. Hence, by employing assumption (1) and (3.15), we have

$$
\begin{aligned}
E\left(\mathcal{F}_{m} \circ v^{m}, \Omega\right) & =\int_{\Omega} \mathscr{L}\left(x, D\left(\mathcal{F}_{m} \circ v^{m}\right)(x)\right) d x \\
& =\int_{\Omega} \mathscr{L}\left(x, D \mathcal{F}_{m}\left(v^{m}(x)\right) D v^{m}(x)\right) d x \\
& \leq \int_{\Omega} \mathscr{L}\left(x, D v^{m}(x)\right) d x+\int_{\Omega} a(x) d x \\
& \leq \int_{\Omega} \mathscr{L}(x, D v(x)) d x+\frac{\alpha}{2}+\|a\|_{L^{1}(\Omega)} \\
& =E(v, \Omega)+\frac{\alpha}{2}+\|a\|_{L^{1}(\Omega)},
\end{aligned}
$$

for $m=m(\alpha)$ large enough. Since $v$ is an $\frac{\alpha}{2}$-minimizer of (3.4) and $v-\mathcal{F}_{m} \circ v^{m} \in$ $\left[W_{0}^{1, q}(\Omega)\right]^{N}$, by choosing $\psi \in\left[W_{0}^{1, q}(\Omega)\right]^{N}$ arbitrary and setting

$$
\phi:=\psi-v+\mathcal{F}_{m} \circ v^{m}
$$

we obtain that $\phi \in\left[W_{0}^{1, q}(\Omega)\right]^{N}$. Thus, by approximate minimality of $v$, we obtain

$$
\begin{aligned}
E(v, \Omega) & \leq E(v+\phi, \Omega)+\frac{\alpha}{2} \\
& =E\left(\mathcal{F}_{m} \circ v^{m}+\psi, \Omega\right)+\frac{\alpha}{2} .
\end{aligned}
$$

By combining (3.16) and (3.18), we see that for any $\alpha>0$, the function $u:=\mathcal{F}_{m} \circ v^{m}$ is an $\left(\alpha+\|a\|_{L^{1}(\Omega)}\right)$-minimizer which in view of (3.13) satisfies (3.6), as desired.

3.2. The general case of integrand which depends on all the arguments. Let us now consider the general functional

$$
E(u, \Omega)=\int_{\Omega} \mathscr{L}(x, u(x), D u(x)) d x .
$$

Hypotheses on the functional (3.19). We shall need to impose the following assumptions: let $\Omega \subseteq \mathbb{R}^{n}$ be open and $\mathscr{L}: \Omega \times \mathbb{R}^{N} \times \mathbb{R}^{N \times n} \longrightarrow \mathbb{R}$ a Carathéodory function such that

(1) $\mathscr{L}\left(x, \eta,_{-}\right)$is almost invariant under the Orthogonal Group, locally uniformly in $\eta$ : for any $R>0$, there exists an $a=a_{R} \in L^{1}(\Omega)$ such that for a.e. $x \in \Omega$, all $|\eta| \leq R$, all $O \in O(N, \mathbb{R})$ and all $P \in \mathbb{R}^{N \times n}$, we have

$$
|\mathscr{L}(x, \eta, P)-\mathscr{L}(x, \eta, O P)| \leq a(x) .
$$

(2) $\mathscr{L}\left(x, \eta,_{-}\right)$is of q-growth: there exist $C>0, q \geq 1, b \in L^{1}(\Omega)$ and $d$ : $\Omega \times \mathbb{R}^{N} \longrightarrow \mathbb{R}$ a Carathéodory function such that

$$
-b(x) \leq \mathscr{L}(x, \eta, P) \leq C|P|^{q}+d(x, \eta) .
$$

(3) There exists a convex set such that the values of $\mathscr{L}\left(x,{ }_{-}, P\right)$ outside of it almost exceed those on the boundary: there exist $l \in L^{1}(\Omega)$ and $\mathcal{C} \subseteq \mathbb{R}^{N}$ compact and convex with $0 \in \operatorname{int}(\mathcal{C})$ such that

$$
\max _{\eta \in \partial \mathcal{C}} \mathscr{L}(x, \eta, P) \leq \inf _{\eta \in \mathbb{R}^{N} \backslash \mathcal{C}} \mathscr{L}(x, \eta, P)+l(x),
$$

for a.e. $x \in \Omega$ and all $P \in \mathbb{R}^{N \times n}$. 
Remark 3.6. Assumptions (1) and (2) are analogous to those of the previous case of (3.4). Assumption (3) says that there exists a convex set $\mathcal{C}$ such that the function $\mathscr{L}\left(x,{ }_{,}, P\right)$ has values on $\mathbb{R}^{N} \backslash \mathcal{C}$ which almost exceed those on $\partial \mathcal{C}$. The nonnegative function $l$ represents the deviation of the restriction $\left.\mathscr{L}\left(x,{ }_{-}, P\right)\right|_{\mathcal{C}}$ from being constant. If $l \equiv 0$, then "almost" in the previous statement can be dropped. Assumption (3) is weaker than requiring $\mathcal{C}$ to be a sublevel set, since the values inside $\mathcal{C}$ may well exceed those on the boundary.

Theorem 3.7 (Folding Principle II). Let $\Omega \subseteq \mathbb{R}^{n}$ be open and $\mathscr{L}: \Omega \times \mathbb{R}^{N} \times$ $\mathbb{R}^{N \times n} \longrightarrow \mathbb{R}$ a Carathédory function satisfying assumptions (1), (2) and (3). Then, for any $\alpha>0$ and any $g \in\left[W^{1, q}(\Omega) \cap L^{\infty}(\Omega)\right]^{N}$ there exists an $\left(\alpha+\|a+l\|_{L^{1}(\Omega)}\right)$ minimizer $u$ of the functional (3.19) in $\left[W_{g}^{1, q}(\Omega)\right]^{N}$ which satisfies

$$
u(\partial \Omega) \subset \mathcal{C} \Longrightarrow u(\Omega) \subseteq \mathcal{C}^{\alpha} .
$$

By Theorem 3.7 we readily obtain the following

Corollary 3.8. In the setting of Theorem 3.7, if moreover $a=l \equiv 0$ in assumptions (1) and (3), then there exists a minimizing family $\left\{u_{\alpha}\right\}_{\alpha>0}$ of problem (1.3) which satisfies as $\alpha \rightarrow 0$ that

$$
u_{\alpha}(\partial \Omega) \subset \mathcal{C} \Longrightarrow u_{\alpha}(\Omega) \subseteq \mathcal{C}^{\alpha} .
$$

Remark 3.9. A statement analogous to that or Remark 3.4 applies here as well. Once again we observe that in general if $a \not \equiv 0$ or $l \not \equiv 0$, there must be an increase at the energy level of the resulting approximate minimizer.

Remark 3.10. The strictness assumption " $u(\partial \Omega) \subset \mathcal{C}$ " appearing in (3.20) and (3.21) can be weakened to " $u(\partial \Omega) \subseteq \mathcal{C}$ " by assuming in addition that the function $\eta \mapsto \mathscr{L}(x, \eta, P)$ has moduli of continuity over compacts depending uniformly in $(x, P) \in \Omega \times \mathbb{R}^{N \times n}$. This is a rather weak requirement which is always satisfied in the decoupled case of $\mathscr{L}(x, \eta, P)=A(x, P)+W(\eta)$, a standard example of which is given by the Action functional $E(u, \Omega)=\int_{\Omega}\left[\frac{1}{2}|D u(x)|^{2}+W(u(x))\right] d x$.

Proof of Theorem 3.7. Fix $\alpha>0$ and let $v \in\left[W_{g}^{1, q}(\Omega)\right]^{N}$ be an $\frac{\alpha}{2}$-minimizer of (3.19) satisfying $v(\partial \Omega) \subset \mathcal{C}$. Let us denote the Folding Maps of $\mathcal{C}$ provided by Theorem 2.1 by $\mathcal{F}_{m}, m \in \mathbb{N}$. Let $\mathcal{P}$ denote the projection map onto $\mathcal{C}$. Let finally $v^{m}$ be the truncate of $v$ given by (3.9) and (3.10). Then, $\mathcal{F}_{m} \circ v^{m}$ is a well defined function in $\left[W^{1, q}(\Omega)\right]^{N}$. By assumption (3), $\mathcal{C}$ is compact. By (3.1) and (3.2), $v(\partial \Omega)$ is closed and since $v(\partial \Omega) \subset \mathcal{C}$, there exists an open neighborhood of $v(\partial \Omega)$ which is contained into $\mathcal{C}$. Hence, $\mathcal{F}_{m}$ leaves the boundary values invariant; we therefore obtain

$$
\left(\mathcal{F}_{m} \circ v^{m}\right)(\partial \Omega)=v(\partial \Omega) .
$$

Consequently, we have that $\mathcal{F}_{m} \circ v^{m} \in\left[W_{g}^{1, q}(\Omega)\right]^{N}$. Again by Theorem 2.1, for $m=m(\alpha)$ large we have

$$
\left(\mathcal{F}_{m} \circ v^{m}\right)(\Omega) \subseteq \mathcal{C}^{\alpha} .
$$

By (3.9), (3.10) and Theorem 2.1, we obtain that

$$
\begin{aligned}
D v^{m} & \longrightarrow D v, \\
\mathcal{F}_{m} \circ v^{m} & \longrightarrow \mathcal{P} \circ v,
\end{aligned}
$$


a.e. on $\Omega$, both along a common subsequence as $m \rightarrow \infty$. By employing the bounds

$$
\begin{aligned}
\left|\mathcal{F}_{m} \circ v^{m}\right| & \leq 2 \operatorname{diam}(\mathcal{C}) \\
|\mathcal{P} \circ v| & \leq \operatorname{diam}(\mathcal{C}), \\
\left|D v^{m}\right| & \leq 2|D v|
\end{aligned}
$$

valid for $m$ large, by the bound of assumption (2) and application of the Dominated Convergence theorem, we obtain

$$
\int_{\Omega} \mathscr{L}\left(x, \mathcal{F}_{m}\left(v^{m}(x)\right), D v^{m}(x)\right) d x \longrightarrow \int_{\Omega} \mathscr{L}(x, \mathcal{P}(v(x)), D v(x)) d x,
$$

as $m \rightarrow \infty$. Now we employ the identity

$$
\begin{aligned}
E\left(\mathcal{F}_{m} \circ v^{m}, \Omega\right) & =\int_{\Omega} \mathscr{L}\left(x,\left(\mathcal{F}_{m} \circ v^{m}\right)(x), D\left(\mathcal{F}_{m} \circ v^{m}\right)(x)\right) d x \\
& =\int_{\Omega} \mathscr{L}\left(x, \mathcal{F}_{m}\left(v^{m}(x)\right), D \mathcal{F}_{m}\left(v^{m}(x)\right) D v^{m}(x)\right) d x
\end{aligned}
$$

in order to estimate the energy $E\left(\mathcal{F}_{m} \circ v^{m}, \Omega\right)$. For, by employing Theorem 2.1, for a.e. $x \in \Omega$, we have that $D \mathcal{F}_{m}\left(v^{m}(x)\right) \in O(N, \mathbb{R})$. We utilize (3.29) and assumption (1) where as $R$ we take

$$
R:=2 \operatorname{diam}(\mathcal{C})
$$

with respective $a=a_{R}$. Then, identity (3.30) implies

$$
\begin{aligned}
E\left(\mathcal{F}_{m} \circ v^{m}, \Omega\right) \leq & \int_{\Omega} \mathscr{L}\left(x, \mathcal{F}_{m}\left(v^{m}(x)\right), D v^{m}(x)\right) d x \\
& +\int_{\Omega} a(x) d x \\
\leq & \int_{\Omega} \mathscr{L}(x, \mathcal{P}(v(x)), D v(x)) d x+\frac{\alpha}{2} \\
& +\|a\|_{L^{1}(\Omega)},
\end{aligned}
$$

for $m=m(\alpha)$ large enough. By assumption (3), a.e. on the set

$$
\{v \notin \mathcal{C}\}:=\left\{x \in \Omega \mid v(x) \in \mathbb{R}^{N} \backslash \mathcal{C}\right\} \subseteq \Omega
$$

we have the estimate

$$
\begin{aligned}
\mathscr{L}\left({ }_{-}, \mathcal{P} \circ v, D v\right) & \leq \max _{\eta \in \partial \mathcal{C}} \mathscr{L}\left({ }_{-}, \eta, D v\right) \\
& \leq \inf _{\eta \in \mathbb{R}^{N} \backslash \mathcal{C}} \mathscr{L}\left(_{-}, \eta, D v\right)+l \\
& \leq \mathscr{L}\left(_{-}, v, D v\right)+l .
\end{aligned}
$$

Trivially, a.e. on its complement $\{v \in \mathcal{C}\}$ we have

$$
\mathscr{L}\left(_{-}, \mathcal{P} \circ v, D v\right)=\mathscr{L}\left(_{-}, v, D v\right)
$$


By employing (3.34) and (3.35), estimate (3.32) implies

$$
\begin{aligned}
E\left(\mathcal{F}_{m} \circ v^{m}, \Omega\right) \leq & \int_{\{v \notin \mathcal{C}\}} \mathscr{L}(x, \mathcal{P}(v(x)), D v(x)) d x \\
& +\int_{\{v \in \mathcal{C}\}} \mathscr{L}(x, \mathcal{P}(v(x)), D v(x)) d x \\
& +\frac{\alpha}{2}+\|a\|_{L^{1}(\Omega)} \\
\leq & \int_{\{v \notin \mathcal{C}\}} \mathscr{L}(x, v(x), D v(x)) d x+\int_{\Omega} l(x) d x \\
& +\int_{\{v \in \mathcal{C}\}} \mathscr{L}(x, v(x), D v(x)) d x \\
& +\frac{\alpha}{2}+\|a\|_{L^{1}(\Omega)} .
\end{aligned}
$$

Hence, by (3.36) we obtain

$$
\begin{aligned}
E\left(\mathcal{F}_{m} \circ v^{m}, \Omega\right) \leq & \int_{\Omega} \mathscr{L}(x, v(x), D v(x)) d x \\
& +\frac{\alpha}{2}+\|a\|_{L^{1}(\Omega)}+\|l\|_{L^{1}(\Omega)} \\
= & E(v, \Omega)+\frac{\alpha}{2}+\|a+l\|_{L^{1}(\Omega)} .
\end{aligned}
$$

Consequently, since $v$ is an $\frac{\alpha}{2}$-minimizer of (3.19) and $v-\mathcal{F}_{m} \circ v^{m} \in\left[W_{0}^{1, q}(\Omega)\right]^{N}$, by choosing $\psi \in\left[W_{0}^{1, q}(\Omega)\right]^{N}$ arbitrary and setting

$$
\phi:=\psi-v+\mathcal{F}_{m} \circ v^{m}
$$

we obtain that $\phi \in\left[W_{0}^{1, q}(\Omega)\right]^{N}$. Thus, by approximate minimality of $v$, we obtain

$$
\begin{aligned}
E(v, \Omega) & \leq E(v+\phi, \Omega)+\frac{\alpha}{2} \\
& =E\left(\mathcal{F}_{m} \circ v^{m}+\psi, \Omega\right)+\frac{\alpha}{2} .
\end{aligned}
$$

Hence, by combining (3.37), (3.39) and (3.23), for any $\alpha>0$ the function $u:=$ $\mathcal{F}_{m} \circ v^{m}$ is an $\left(\alpha+\|a+l\|_{L^{1}(\Omega)}\right)$-minimizer satisfying property (3.20). Theorem 3.7 follows.

Acknowledgement. I thank L. Ambrosio, J. Ball, P. Bates, B. Dacorogna, L.C. Evans, F. Leonetti, and A. Tertikas for their suggestions and comments on an earlier weaker version of this manuscript which led to substantial improvements.

Last but not least, I am profoundly indebted to N. Alikakos for his constructive criticism and for our inspiring scientific discussions.

\section{REFERENCES}

[AG] S. Alexander, M. Ghomi, The convex hull property and topology of hypersurfaces with nonnegative curvature, Advances in Mathematics Volume 180, Issue 1, 2003, 324 - 354.

[AK] N. Alikakos, N. Katzourakis, Heteroclinic Travelling Waves of Gradient Diffusion Systems, Trans. Amer. Math. Soc. 363 (2011), 1365 - 1397.

[B] J. Ball, private conversation.

[BF1] M. Bildhauer, M. Fuchs, Partial regularity for a class of anisotropic variational integrals with convex hull property, Asymptotic Analysis 32 (2002) 293 - 315. 
[BF2] M. Bildhauer, M. Fuchs, A geometric maximum principle for variational problems in spaces of vector valued functions of bounded variation, Zap. Nauchn. Sem. POMI, 2010, 385, 5 17 .

[CM] T. H. Colding, W. P. Minicozzi II, An excursion into geometric analysis, Surv. Differ. Geom., IX, Int. Press, Somerville, MA, (2004).

[D1] B. Dacorogna, Direct Methods in the Calculus of Variations, Applied Mathematical Sciences 78, Springer - Verlag, 2008 edition.

[D2] B. Dacorogna, Some geometric and algebraic properties of various types of convex hulls, in "Nonsmooth mechanics and Analysis: Theoretical and Numerical advances", Advances in Mechanics and Mathematics, Springer, (2006), 25 - 34.

[D3] B. Dacorogna, Non convex problems of the calculus of variations and differential inclusions, in Handbook of Differential Equations (Stationary PDEs) Volume 2, Elsevier, North Holland, 2 (2005), 57 - 126.

[DF] B. Dacorogna, A. Ferriero, Regularity and Selecting Principles for Implicit Ordinary Differential Equations, Disc. Cont. Dynamical Systems, B, 11 (2009), 87 - 101.

[DPR] B. Dacorogna, G. Pisante, A. M. Ribeiro, On non quasiconvex problems of the calculus of variations; Discrete and Continuous Dynamical Systems, Series A 13 (2005), 961 - 983.

[DLM] D'Ottavio, A., Leonetti, F., Musciano, C., Maximum principle for vector valued mappings minimizing variational integrals, Atti Sem. Mat. Fis. Uni. Modena XLVI (1998), 677 - 683.

[Fr] L. E. Fraenkel, An Introduction to Maximum Principles and Symmetry in Elliptic Problems, Cambridge Tracts in Mathematics 128, 2000.

[Ga] F. Gazzola, Existence of Minima for Nonconvex Functionals in Spaces of Functions Depending on the Distance from the Boundary, Arch. Rational Mech. Anal. 150 (1999) 57 76.

[Gi] E. Giusti, Direct Methods in the Calculus of Variations, World Sceintific, 2005.

[L1] F. Leonetti, Maximum principle for vector-valued minimizers of some integral functionals, Boll. Un. Mat. Ital. 5-A (1991), 51 - 56.

[L2] F. Leonetti, Maximum principle for functionals depending on minors of the jacobian matrix of vector-valued mappings, Centre for Mathematical Analysis, Australian National Univ., Research Report n.20, 1990.

[LS] F. Leonetti, F. Siepe, Maximum Principle for Vector Valued Minimizers, Journal of Convex Analysis 12 (2005) $267-278$.

[O1] R. Osserman, The Convex Hull Property of Immersed Manifolds, J. Diff. Geom. 6 (1971), $267-270$.

[O2] R. Osserman, A Survey of Minimal Surfaces, 2nd edition, Dover, New York, 1986.

[PS] P. Pucci, J. Serrin, The Maximum Principle, Progress in Nonlinear Differential Equations and Applications 73, Birkhäuser, 2007.

BCAm - Basque Center for Applied Mathematics, Mazarredo 14, Bilbao, E-48009, SPAIN

E-mail address: nkatzourakis@bcamath.org 